\title{
A New Comprehensive Catalogue of Infrared Dark Clouds
}

\author{
G. A. Fuller ${ }^{1}$ and N. Peretto ${ }^{1}$ \\ ${ }^{1}$ Jodrell Bank Centre for Astrophysics, School of Physics \& Astronomy, Alan Turing Building, \\ University of Manchester, Oxford Road, Manchester, M13 9PL \\ email: G.Fuller@manchester.ac.uk
}

\begin{abstract}
To better characterise infrared dark clouds (IRDCs), and the star formation within them, a comprehensive catalogue of IRDCs has been constructed from the Spitzer GLIMPSE and MIPSGAL archival data. Mosaicing the individual survey blocks together, we have used a new extraction method to identify dark clouds up to $30^{\prime}$ in size, and produce a column density image of each cloud. In total the catalogue contains over 11,000 clouds, defined as connected regions with 8 micron optical depth $>0.35$ (corresponding to column densities $>10^{22} \mathrm{~cm}^{-2}$ ). The extraction algorithm also identifies sub-structures (fragments) within each cloud. These Spitzer dark clouds (SDCs) range in mass from $10 \mathrm{M}_{\odot}$ to $10^{4} \mathrm{M}_{\odot}$. About $80 \%$ of the SDCs were previously unidentified. Only $\sim 30 \%$ of the SDCs are associated with $24 \mu \mathrm{m}$ point-like sources, leaving the majority of these clouds with no apparent sign of star formation activity. This new catalogue provides an important new resource for future studies of the initial conditions of star formation in the Galaxy.
\end{abstract}

Keywords. Star formation, Molecular clouds

Infrared dark clouds seen in absorption against extended emission were first identified by Pérault et al. (1996) using ISO, and subsequently an extensive catalogue was produced from the MSX Galactic Plane survey (Simon et al. 2006). Using the Spitzer GLIMPSE and MIPSGAL data we have recently constructed a new catalogue of over 11,000 dark clouds identified from their $8 \mu \mathrm{m}$ absorption using a new extraction algorithm which also identifies and characterises fragments within the clouds (Peretto \& Fuller 2009). This catalogue, including a map of each cloud, is available online at www.irdarkclouds.org. The majority of these Spitzer dark clouds (SDCs) were previously unidentified, with only $20 \%$ of them appearing in the earlier MSX-based IRDC catalogue. Compared with the MSX clouds, the SDCs are more concentrated towards the Galactic Plane.

Inferring the mass of the clouds from their extinction and adopting a typical distance to a cloud of $4 \mathrm{kpc}$, the mass distribution of the clouds is found to be a power-law with an index indistinguishable from that for the mass distribution of CO clumps in molecular clouds (e.g. Kramer et al. 1998). However, the mass distribution for the fragments within the clouds has a log-normal form, indicative of a turbulent origin for these sub-structures (Hennebelle \& Chabrier 2008).

\section{References}

Hennebelle, P. \& Chabrier, G. 2008, ApJ 684, 395

Kramer, C., Stutzki, J., Rohrig, R., \& Corneliussen, U. 1998, A\&̈A 329, 249

Pérault, M., Omont, A., Simon, G., et al. 1996, A\&A 315, 165

Peretto, N. \& Fuller, G. A. 2009, A\&A in press

Simon, R., Rathborne, J. M., Shah R. Y., Jackson, J. M., \& Chambers, E. T. 2006, ApJ 653, 1325 